\title{
CATMAG : les énergies renouvelables à portée de main
}

Grâce à des nanoparticules chauffées par induction magnétique et utilisées comme catalyseur, Bruno Chaudret, au Laboratoire de Physique et Chimie des Nano-Objets (a), à Toulouse, propose un procédé original pour la synthèse de méthane à partir de dioxyde de carbone et de dihydrogène.

Des bouteilles contenant différents réactifs gazeux, un réacteur en verre enchâssé dans une bobine magnétique directement relié à un chromatographe en phase gazeuse et un spectromètre de masse, le tout piloté par un ordinateur... "Désormais, nous allons pouvoir passer à la phase d'optimisation ", s'enthousiasme Bruno Chaudret, porteur du projet CATMAG, lauréat du premier programme d'incitation à l'innovation du CNRS, piloté par l'INP. Objectif : mettre sur la voie de l'industrialisation un nouveau procédé de catalyse à base de nanoparticules chauffées par induction magnétique, dans le but de stocker des énergies renouvelables intermittentes sous la forme de méthane synthétisé à partir de dioxyde de carbone et de dihydrogène.

De façon amusante, lorsque, 25 ans plus tôt, le chimiste se lance dans l'art de façonner des nano-objets organométalliques en solution, il n'est nullement question d'applications. "L'enjeu, purement fondamental, était alors la synthèse de nanoparticules, c'est-à-dire de parvenir à contrôler des réactions chimiques à une échelle mésoscopique jusqu'alors inexplorée ", se souvient-il.

Mais au début des années 2000, Bruno Chaudret entrevoit ainsi la possibilité de façonner des nanoparticules magnétiques pour des applications, notamment en biologie. "Placées dans un champ magnétique haute fréquence, ces particules ont la propriété de chauffer considérablement. Ainsi, nous pensions par exemple, les utiliser pour tuer des cellules cancéreuses ", explique le scientifique.

L'astuce ? Avoir imaginé un procédé pour contourner le problème d'oxydation de la surface de ces nano-objets en les habillant d'une coquille de carbure de fer. Le succès est tel que B. Chaudret cherche et trouve une formulation pour produire dix fois plus de chaleur que le procédé actuel.

(a) Laboratoire de Physique et Chimie des Nano-Objets (LPCNO), UMR 5215 CNRS/INSA Toulouse/Université Toulouse III Paul Sabatier. L'activité de recherche du LPCNO se focalise autour de la synthèse chimique, de la caractérisation et de la modélisation de nano-objets. Les objets synthétisés sont caractérisés par différentes méthodes physiques (magnétisme, mesures optiques, etc.) et font l'objet de modélisations théoriques. Ces travaux de recherche présentent des applications directes dans les domaines de la microélectronique, de l'optoélectronique ainsi que de la médecine, notamment en oncologie.

(b) Société LEAF, Ecoparc Technowest, 17 rue du Commandant Charcot, 33290 Blanquefort. Contact : leaf.energie@gmail.com

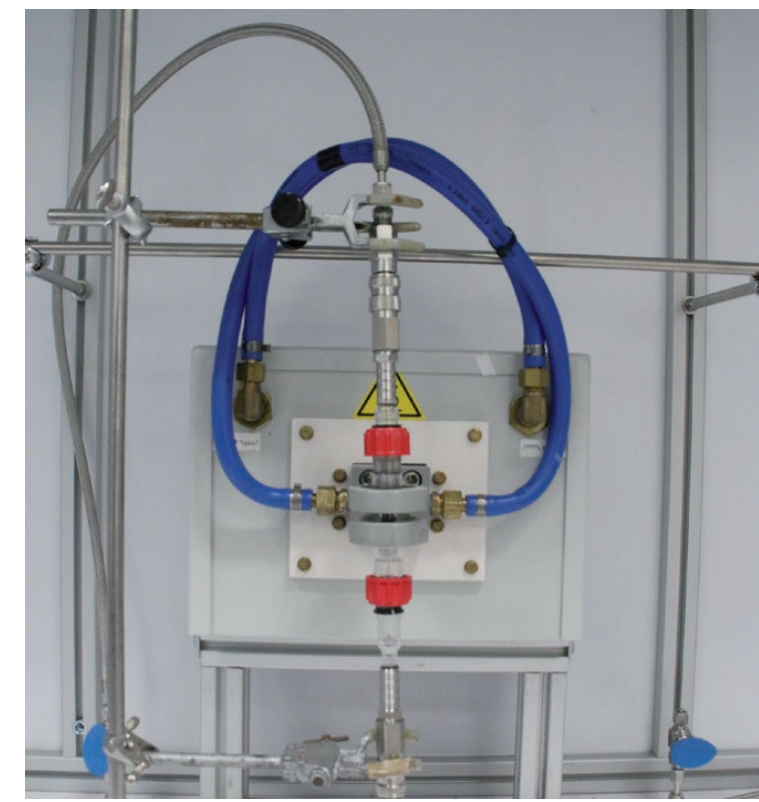

Dispositif de catalyse par induction magnétique, composé d'un four dans lequel est placé le catalyseur et d'une bobine à induction permettant le chauffage magnétique des nanocatalyseurs.

De là, l'idée d'utiliser ces particules pour la catalyse de réactions chimiques, sans avoir à chauffer l'ensemble du milieu réactionnel. Conséquence : une synthèse à moindre coût, mais également ultraflexible en matière d'utilisation, car une milliseconde suffit à chauffer les particules en présence d'un champ magnétique.

Deux brevets plus tard, la technologie conçue par Bruno Chaudret est donc entrée en phase de développement, en collaboration avec la société $\operatorname{LEAF}^{(\mathrm{b})}$. Cette entreprise envisage, à échéance de quelques années, de commercialiser là où le gaz de ville n'est pas accessible, de petites installations capables de fournir du méthane, synthétisé à partir de $\mathrm{CO}_{2}$ capturé à la sortie des cheminées et d'hydrogène obtenu par électrolyse de l'eau grâce à l'énergie solaire. "On ne va pas sauver la planète, juge modestement Bruno Chaudret. Mais il n'est pas exclu que cela marche!"

Mathieu Grousson, journaliste

- Contact chercheur

Bruno Chaudret (chaudret@insa-toulouse.fr)

Directeur de recherche au CNRS. 